\title{
Co,jF- $9706289--$ \\ Identification of a Reflection Boundary Coefficient in an Acoustic Wave Equation by Optimal Control Techniques *
}

\author{
Suzanne Lenhart ${ }^{1,2}$, Vladimir Protopopescu ${ }^{2}$, Jiongmin Yong ${ }^{3}$ \\ ${ }^{1}$ University of Tennessee \\ Mathematics Department \\ Knoxville, Tennessee 37996-1300 \\ ${ }^{2}$ Oak Ridge National Laboratory \\ Computer Science and Mathematics Division \\ Oak Ridge, Tennessee 37831 \\ ${ }^{3}$ Fudan University \\ Department of Mathematics \\ Shanghai, 200433 China
}

\begin{abstract}
"This submitted manuscript has been authored by a contractor of the U. S. government under Contract No. DE-AC05-98OR22464. Accordingly, the U. S. Government retains a nonexclusive, royalty-free license to publish or reproduce the published form of this contribution, or allow others to do so, for U. S. Government purposes."
\end{abstract}

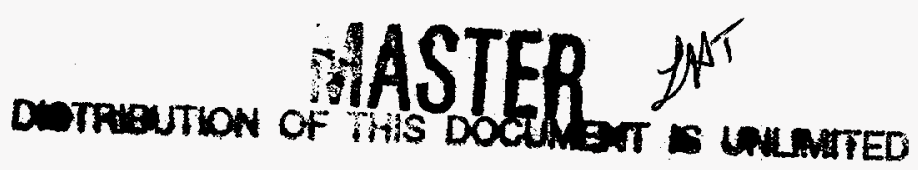

\footnotetext{
*Research supported by the U. S. Department of Energy, Office of Basic Energy Sciences, under contract No. AC05-96OR22464 with Lockheed Martin Energy Research Corporation.
} 


\section{DISCLAIMER}

This report was prepared as an account of work sponsored by an agency of the United States Government. Neither the United States Government nor any agency thereof, nor any of their employees, makes any warranty, express or implied, or assumes any legal liability or responsibility for the accuracy, completeness, or usefulness of any information, apparatus, product, or process disclosed, or represents that its use would not infringe privately owned rights. Reference herein to any specific commercial product, process, or service by trade name, trademark, manufacturer, or otherwise does not necessarily constitute or imply its endorsement, recommendation, or favoring by the United States Government or any agency thereof. The views and opinions of authors expressed herein do not necessarily state or reflect those of the United States Government or any agency thereof. 


\section{DISCLAIMER}

Portions of this document may be illegible in electronic image products. Images are produced from the best available original document. 


\title{
IDENTIFICATION OF A REFLECTION BOUNDARY COEFFICIENT IN AN ACOUSTIC WAVE EQUATION BY OPTIMAL CONTROL TECHNIQUES
}

\author{
SUZANNE LENHART ${ }^{1,2}$ \\ VLADIMIR PROTOPOPESCU ${ }^{2}$ \\ JIONGMIN YONG ${ }^{3}$
}

\begin{abstract}
We apply optimal control techniques to find approximate solutions to an inverse problem for the acoustic wave equation. The inverse problem (assumed here to have a solution) is to determine the boundary reflection coefficient from partial measurements of the acoustic signal. The sought reflection coefficient is treated as a control and the goal - quantified by an approximate functional - is to drive the model solution close to the experimental data by adjusting this coefficient. The problem is solved by finding the optimal control that minimizes the approximate functional. Then by driving the "cost of the control" to zero one proves that the corresponding sequence of optimal controls represents a converging sequence of estimates for the solution of the inverse problem. Compared to classical regularization methods (e.g., Tikhonov coupled with optimization schemes), our approach yields: (i) a systematic procedure to solve inverse problems of identification type and (ii) an explicit expression for the approximations of the solution.
\end{abstract}

\section{Introduction}

Over the last two decades, parameter identification, i.e. reconstruction of model properties from observed data has become one of the most active and work intensive areas of applied mathematics. Traditionally, identification techniques have been associated to model (retro)fitting and validation, reverse engineering, and signal detection and interpretation. With the advent of high power computers, new standards of performance have become the

\footnotetext{
${ }^{1}$ University of Tennessee, Mathematics Department, Knoxville, TN 37996-1300

${ }^{2}$ Oak Ridge National Laboratory, Computer Science and Mathematics Division, Oak Ridge, TN 378316364

${ }^{3}$ Fudan University, Department of Mathematics, Shanghai, 200433 China
}

Typeset by $\mathcal{A} M \mathcal{S}-\mathrm{TEX}_{\mathrm{E}}$ 
required norm in modern fields such as communication systems, computer networks, astrophysics, bioengineering, or sophisticated military command and control systems. These standards, especially in domains where on-line responses are crucial, make it necessary to have a much better understanding of the quantitative models involved and warrant the continuing interest in the development of new robust and rigorous identification methods.

¿From a formal mathematical standpoint, the parameter identification problem is an inverse problem that consists of two separate albeit related subproblems, namely: (a) the identifiability problem and (b) the parameter estimation problem. To understand more precisely the nature of these inverse problems we first describe briefly the direct problem. In the direct problem, a physical system is described (modeled) by the state function, $u$, which satisfies the abstract operator state equation with data $F$ :

$$
A(u(\xi) ; F(\xi))=0 .
$$

The state of the system and the data may be scalar or vector. The independent variables of the problem, $\xi$ - which for evolution problems contain the time, $t$ - take values in the domain $\Omega \subset \mathrm{R}^{n}$, with sufficiently regular boundary, $\partial \Omega$. The operator $A$ may be rather general, including nonlinear equations of higher order, ordinary differential equations, hybrid systems, etc.

If the system (1.1) accurately describes a realistic physical situation, the direct problem is well-posed, i.e. the data $F$ (parameters, functions, coefficients, sources, initial and boundary values, etc.) determine uniquely a regular solution $u[4,14]$. The inverse problem is usually ill-posed in the sense that existence, uniqueness, and/or regularity cannot be expected in general $[1,5,8]$.

The identifiability problem consists in studying the well-posedness of the parameter identification problem. Identifiability is loosely defined as the injectivity of the mapping between the sought parameter and ther output within the used model that is supposed to be completely and accurately known. The identifiability problem consists in determining whether one can uniquely recover (a part of) $F$ from the model solution, $u$. The parameter estimation problem consists in finding an estimated value of the unknown parameter from the data within the admissible set that is consistent with identifiability. In the following, we shall assume that the parameters are uniquely identifiable. The remaining problem is then related to regularity, explicit characterization, and approximation of the parameters.

More precisely, we shall tackle the following identification problem corresponding to the direct problem (1.1): "Given partial observations, $B \tilde{u}$, of the true solution $\tilde{u}$, in a subdomain $\Omega^{\prime}$ of the phase space, $\Omega^{\prime} \subset \Omega$, and a known part, $\phi$, of the data $F$, we seek to determine the unknown part of the data, $f . "$

The standard methods of solving parameter estimation problems are based on Tikhonov's regularization. In this approach, one constructs - starting from actual observations, $B \tilde{u}$, an approximate functional:

$$
J_{\beta}(f)=\frac{1}{2}\|B u-B \tilde{u}\|^{2}+\frac{\beta}{2}\|f\|^{2}, \quad \beta>0
$$


where $u$ is the solution of $(1.1)$ for the data $F=(\phi, f)$. The exact form of the cost functional and the types of norms involved depend on the concrete problem that has to be solved. Tikhonov's approach [28] and its variants seek to minimize the functional $J_{\beta}(f)$ - for a fixed $\beta$ - over the set of unknown data. It assures - in principle - that the observation $B u$ of the model solution, $u(f)$, obtained with parameter $f$, will approach the actual observation, $B \tilde{u}$. The functions $f_{\beta}$ which achieve the absolute minimization of $J_{\beta}(f)$ represent the approximate solution of the parameter identification problem. The disadvantages of this approach are:

- when $\beta$ is small, the problem is unstable;

- when $\beta$ is large, the solution is not accurate;

- there is no systematic procedure for finding the absolute minimum;

- there are no systematic means to evaluate the approximations.

To eliminate most of the disadvantages above, we propose a different approach to the inverse problem of identification, based on optimal control for operator equations as developed by J.-L. Lions $[17,20]$.

Our idea is to consider a family of functionals (1.2) for $\beta \geq 0$. For each $\beta$ strictly positive one considers the unknown data, $f$, as a control which belongs to a certain bounded set, $U$; the control has to be adjusted in order to minimize the functional $J_{\beta}(f)$. The minimum of the cost functional over $f$ is attained at the optimal control, $f=f_{\beta}$ :

$$
J_{\beta}\left(f_{\beta}\right)=\inf _{f \in U} J_{\beta}(f) \text {. }
$$

Letting the sequence of $\beta$ tend toward zero, one can verify that the sequence $f_{\beta}$ converges in an appropriate sense to an element of the control set, $f^{*} \in U$. This element represents the (sometimes unique) solution of the parameter identification problem.

In this paper, we shall specifically apply the formalism to a hyperbolic (acoustic wave) equation. The paper is structured as follows. In Section 3, we shall briefly review the existence of a weak solution to the state problem stated in Section 2, and of an optimal control (for a more detailed analysis see Ref 12). In Section 4, we derive the necessary conditions that an optimal control and its corresponding state must satisfy. Uniqueness of the optimal control for sufficiently short times is proved in Section 5. Section 6 illustrates the application of this optimal control problem to determining an estimate of the reflection coefficient from partial measurements of the solution.

\section{Statement of the Problem}

Given a bounded domain $D \subset \mathrm{R}^{2}$ with $C^{1}$ boundary, define the spatial domain

$$
\Omega=\{(x, y, z) \mid(x, y) \in D, u(x, y)<z<0\},
$$

where $u: D \rightarrow(-\infty, 0)$ is a $C^{2}$ function. Assume the region $\Omega$ contains a certain medium (like water in a section of the ocean) with a known velocity tensor $E$. Let $K>0$ be a finite constant and define the control set

$$
U_{K}=\left\{\sigma \in L^{\infty}(D) \mid 0 \leq \sigma(x, y) \leq K\right\}
$$


Given a control $\sigma \in U_{K}$, we consider the solution $w=w(\sigma)$ of the acoustic wave equation:

$$
\begin{aligned}
& w_{t t}-\nabla(E \nabla w)=f \quad \text { in } Q \\
& w=0 \quad \text { on } \Sigma \times(0, T), \quad \text { sides of spatial domain } \\
& \frac{\partial w}{\partial \nu}=0 \quad \text { on } D \times\{z=0\} \times(0, T), \quad \text { top of spatial domain } \\
& \frac{\partial w}{\partial \nu}+\sigma w=0 \quad \text { on } \Gamma \times(0, T), \quad \text { bottom of spatial domain } \\
& w=g_{1}, w_{t}=g_{2} \quad \text { on } \Omega \times\{0\},
\end{aligned}
$$

where

$$
\begin{aligned}
& \Gamma=\{(x, y, u(x, y)) \mid(x, y) \in D\} \\
& \Sigma=\{(x, y, z) \mid(x, y) \in \partial D, u(x, y)<z<0\} \\
& Q=\Omega \times(0, T)
\end{aligned}
$$

and

$$
\frac{\partial w}{\partial \nu}=E \nabla w \cdot \eta \quad \text { with } \eta \text {, the outward unit normal vector at the boundary. }
$$

The objective functional $J_{\beta}(\sigma)$ is defined by

$$
J_{\beta}(\sigma)=\frac{1}{2} \int_{G \times(0, T)}(w-h)^{2} d x d y d z d t+\frac{\beta}{2} \int_{\Gamma}(\sigma(x, y))^{2} d s
$$

where $G \subset \Omega$ with positive measure. The first term in $J_{\beta}(\sigma)$ drives $w$ close to the target $h$ on $G \times(0, T)$ and the second term is the cost of the control. We seek to characterize $\sigma_{\beta}$ such that

$$
J_{\beta}\left(\sigma_{\beta}\right)=\inf _{\sigma \in U_{K}} J_{\beta}(\sigma) .
$$

After this characterization has been completed, we let the parameter $\beta$ go to zero to approximate a solution to an inverse problem. The inverse problem is to identify $\sigma$ from observations $h$ of a solution $w$ on $G \times(0, T)$, resulting from a signal source $f$. Assuming that this inverse problem has a unique solution, then for $\beta$ small, the optimal control determined from $J_{\beta}, \sigma_{\beta}$, will approximate it reasonably well.

This bilinear optimal control problem is new for wave equations. See [3] for a controllability result for wave equation using a velocity damped control term. See [14] for a similar bilinear boundary optimal control problem in the parabolic case.

The approach of using optimal control techniques with adjoint equations to approximate solutions to inverse problems of identification type is different from traditional approaches which couple Tikhonov regularization (with a functional like ours) with an optimization 
algorithm [1]. Our approach has the advantage of an explicit characterization of the approximation $\sigma_{\beta}$. In a series of recent papers $[21,22,29]$, Puel and Yamamoto obtain uniqueness, stability, and reconstruction results for the inverse problem of identifying the source in a wave equation. Their work is based on exact controllability results and the Hilbert Uniqueness Method [18,19,20] that apply to linear equations only. Moreover, the identification is realized only for special types of observed data.

The other approaches which use control theory for solving inverse problems are limited to particular cases. Indeed, Russell [7, 23-26] developed the control theory for the wave equation, including several controllability and stability results. However, our framework is applicable to more general equations. Lasiecka and Triggiani $[10,11]$ further extended control theory for wave equations, in particular developing better trace estimates and the Riccati equation framework for linear-quadratic problems. Our control problem cannot be treated within this scheme since it is bilinear - $\sigma w$ - in the boundary condition. Liang [15] analyzes a simpler bilinear optimal control problem for a wave equation with the control in the state equation itself.

Finally, ideas more closely related to the present approach have been developed by Tikhonov [28], Chavent [4], James and Sepulveda [9] and by the authors $[12,13]$.

Tikhonov introduces the regularization to stabilize an optimal control problem where the objective functional does not depend explicitly on the control [28].

Chavent [4] has investigated the stability of applying optimal control techniques to identification problems and conditions to guarantee the identifiability of the parameters. Barbu and Pavel [2] recently solved an optimal control problem, which approximates the inverse problem of identifying the acoustic impedance function in a one dimensional wave equation.

James and Sepulveda [9] solve the parameter identification problem by treating it as a constrained optimization problem. They formally tackle the latter by the Lagrangian method and solve it numerically without establishing any rigorous result related to its solvability.

The results contained in this paper further the application of our general formalism [13] to the parameter identification problem for the acoustic wave equation [12].

\section{Existence of an Optimal Control}

To define the solution space for the state problem (2.1), let

$$
V=\left\{v \in H^{1}(\Omega) \mid v=0 \text { on } \Sigma\right\}
$$

with norm

$$
\|v\|_{V}=\left(\int_{\Omega}|\nabla v|^{2} d x d y d z\right)^{\frac{1}{2}}
$$

Note that this norm on $V$ is equivalent to the usual $H^{1}$ norm due to zero boundary 
conditions on $\Sigma$ and Poincaré's inequality. We make the following assumptions:

$$
\begin{aligned}
& E \in C\left(\bar{\Omega} ; \mathrm{R}^{3 \times 3}\right) \text { and } E(x, y, z) \geq \delta I \\
& \quad \text { for all }(x, y, z) \in \bar{\Omega} \text {, for some } \delta>0 \\
& f, f_{t} \in L^{2}(Q) \\
& G \subset \Omega \text { with positive Lebesgue measure } \\
& h \in L^{2}(G \times(0, T)) \\
& u \in C^{2}(D), \quad u(x, y)<0 \\
& g_{1} \in H^{2}(\Omega) \cap V, \quad g_{2} \in H^{1}(\Omega) .
\end{aligned}
$$

We introduce the bilinear form:

$$
B[w, v ; t]=\int_{\Omega} E \nabla w \cdot \nabla v(x, y, z, t) d x d y d z+\int_{\Gamma} \sigma w v(x, y, u(x, y), t) d s
$$

for $w, v \in L^{2}(0, T ; V), 0 \leq t \leq T$.

Definition: A function $w \in L^{2}(0, T ; V)$ with $w_{t} \in L^{2}\left(0, T ; L^{2}(\Omega)\right)$ and $w_{t t} \in L^{2}\left(0, T ; V^{\prime}\right)$ is a weak solution of $(2.1)$ if

(i) $\left\langle w_{t t}, v\right\rangle+B[w, v ; t]=\int_{\Omega} f(t) v d x d y d z$ for each $v \in V$ and for a.e. $0 \leq t \leq T$,

(ii) at $t=0, w=g_{1}, w_{t}=g_{2}$.

Here $\langle\cdot, \cdot\rangle$ denotes the $V^{\prime}, V$ duality pairing. Note that we also have $w \in C\left([0, T] ; L^{2}(\Omega)\right)$ and $w_{t} \in C\left([0, T] ; V^{\prime}\right)$ (see $[3]$ ), so (ii) makes sense.

The weak solution to the state problem (2.1) is constructed by applying Galerkin's method of finite dimensional approximations [6]. Unlike alternative methods used to prove existence here (e.g. semigroups), the Galerkin method clearly displays the regularity and the dependence on $\sigma$. The detailed derivation of these results is contained in [12].

Theorem 3.1. For $\sigma \in U_{K}$, the state problem has a unique weak solution $w$ that satisfies the following regularity estimate:

$$
\sup _{0 \leq t \leq T}\left(\|w(t)\|_{V}+\left\|w_{t}(t)\right\|_{L^{2}(\Omega)}\right)+\left\|w_{t t}\right\|_{L^{2}\left(0, T ; V^{\prime}\right)} \leq C\left(\left\|g_{1}\right\|_{V}+\left\|g_{2}\right\|_{L^{2}(\Omega)}+\|f\|_{L^{2}(Q)}\right) .
$$

Sketch of the Proof. One considers a sequence of approximations $w_{n}$ to the solution $w$

$$
w_{m}(t)=w_{m}(x, y, z, t)=\sum_{k=1}^{m} d_{k}^{m}(t) \psi_{k}, m \in \mathrm{N}
$$

$\left\{\psi_{k}\right\}_{k \in \mathbb{N}}$ is a basis for $V$ 
and

$\left\{\psi_{k}\right\}_{k \in \mathbb{N}}$ is an orthonormal basis of $L^{2}(\Omega)$,

where $\left\{\psi_{k}\right\}$ are eigenvectors (corresponding to the eigenvalue $\lambda_{k}$ ) of the following SturmLiouville problem:

$$
\begin{aligned}
& \frac{\partial \psi}{\partial \nu}+\sigma \psi=0 \quad \text { on } \Gamma \\
& \psi=0 \text { on } \Sigma \\
& \frac{\partial \psi}{\partial \nu}=0 \quad \text { on } D \times\{z=0\}
\end{aligned}
$$

The functions $d_{k}^{m}(t)$ satisfy

$$
d_{k}^{m \prime \prime}(t)+\sum_{\ell=1}^{m} B\left[w_{\ell}, w_{k} ; t\right] d_{\ell}^{m}(t)=\int_{\Omega} f(t) w_{k} d \Omega
$$

and

$$
d_{k}^{m}(0)=\left(g_{1}, \psi_{k}\right)_{L^{2}}, \quad d_{k}^{m \prime}(0)=\left(g_{2}, \psi_{k}\right)_{L^{2}}, \quad k=1, \ldots, m .
$$

Using the orthogonality of $\left\{\psi_{k}\right\}$ in $L^{2}(\Omega)$ and the definition of $w_{m}$, one shows that $w_{m}$ approximately satisfies the definition of the weak solution for $v=\psi_{k}$ and thus for $v=w_{m}$. One can then derive a priori estimates for $w_{m}$ from which, by passing to the limit, one obtains convergences on subsequences. In turn, these convergences are sufficient to show that the limit $w$ of the sequence $w_{m}$ is the unique weak solution of the equation (2.1). $\quad$ a

Additional regularity on the state solution is needed to derive the necessary conditions that an optimal control and corresponding state must satisfy. In fact, one can prove [12]:

Proposition 3.2. The weak solution of (2.1) has additional regularity:

$$
\sup _{0 \leq t \leq T}\left[\int_{\Omega}\left(w_{t t}\right)^{2}(x, y, z, t) d \Omega+\int_{\Omega}\left|\nabla w_{t}\right|^{2}(x, y, z, t) d \Omega+\int_{\Gamma} \sigma\left(w_{t}\right)^{2}(x, y, u(x, y), t) d s\right] \leq C
$$

with $C$ depending only on $\|f\|_{L^{2}},\left\|f_{t}\right\|_{L^{2}},\left\|g_{1}\right\|_{H^{2}}$ and $\left\|g_{2}\right\|_{H^{1}}$.

Remarks: 1 . We do not get the $L^{2}\left(0, T ; H^{2}(\Omega)\right)$ estimate on $w$ since we have a Robin boundary condition on the bottom with only $L^{\infty}$ regularity on the coefficient $\sigma$. See [6] for this estimate for Dirichlet boundary conditions.

2. Note that we also obtain

$$
\sup _{0 \leq t \leq T} \int_{\Gamma} w_{t}^{2}(x, y, u(x, y), t) d s \leq C
$$


from the bound on

$$
\int_{\Omega}\left|\nabla w_{t}\right|^{2}(x, y, z, t) d \Omega
$$

and Poincaré's Inequality, since

$$
\begin{aligned}
\int_{\Gamma}\left(w_{t}\right)^{2}(t) d s & \leq C \int_{\Omega}\left|\nabla w_{t}\right|^{2}(t) d \Omega+\int_{\Omega}\left(w_{t}\right)^{2}(t) d \Omega \\
& \leq C_{1} \int_{\Omega}\left|\nabla w_{t}\right|^{2}(t) d \Omega
\end{aligned}
$$

and $w_{t}=0$ on $\Sigma \times\{t\}$.

Based on the existence and regularity results contained in Theorem 3.1 we can now prove the existence of an optimal control.

Theorem 3.3: There exists a control $\sigma_{\beta}$ in $U_{K}$ that minimizes the functional $J_{\beta}(\sigma)$ over $U_{K}$.

Proof. Let $\left\{\sigma^{n}\right\}$ be a minimizing sequence, i.e.

$$
\lim _{n \rightarrow \infty} J_{\beta}\left(\sigma^{n}\right)=\inf _{\sigma \in U_{K}} J_{\beta}(\sigma)
$$

Let $w^{n}=w\left(\sigma^{n}\right)$ be the corresponding state solution. By estimates in Theorem 3.1,

$$
\left\|w^{n}\right\|_{L^{2}(0, T, V)}+\left\|w_{t}^{n}\right\|_{L^{2}\left(0, T ; L^{2}(\Omega)\right)}+\left\|w_{t t}^{n}\right\|_{L^{2}\left(0, T, V^{\prime}\right)} \leq C_{1}
$$

where the constant $C_{1}$ is independent of $n$. Then there exists $\sigma_{\beta} \in U_{K}, w_{\beta} \in L^{2}(0, T ; V)$ such that, on a subsequence,

$$
\begin{aligned}
& w^{n} \rightarrow w_{\beta} \text { weakly in } L^{2}(0, T ; V) \\
& w_{t t}^{n} \rightarrow\left(w_{\beta}\right)_{t t} \text { weakly }{ }^{*} \text { in } L^{2}\left(0, T ; V^{\prime}\right)
\end{aligned}
$$

and

$$
\sigma^{n} \rightarrow \sigma_{\beta} \text { weakly on } L^{2}(\Gamma) .
$$

Using a compactness result [27],

$$
w^{n} \rightarrow w_{\beta} \text { strongly in } L^{2}\left(0, T, H_{\{0\}}^{\frac{1}{2}+\varepsilon}(\Omega)\right)
$$

where the subscript $\{0\}$ means zero on $\partial D$, and $0<\varepsilon<\frac{1}{2}$. By trace results,

$$
w^{n} \rightarrow w_{\beta} \text { strongly in } L^{2}(0, T ; \partial \Omega),
$$


which is needed for convergence of the term

$$
\int_{\Gamma \times(0, T)} \sigma^{n} w^{n} \phi d s
$$

in the weak form of solution $w^{n}$. Thus we can pass to the limit and conclude that $w_{\beta}=$ $w\left(\sigma_{\beta}\right)$.

The objective functional $J_{\beta}(\sigma)$ is lower semi-continuous with respect to $L^{2}$ weak convergence and hence the minimum of $J_{\beta}(\sigma)$ is attained by $\sigma_{\beta}$. a

\section{Necessary Conditions}

To derive necessary conditions that an optimal pair $\sigma_{\beta}, w_{\beta}=w\left(\sigma_{\beta}\right)$ must satisfy, we differentiate the map

$$
\sigma \rightarrow J_{\beta}(\sigma)
$$

with respect to $\sigma$. Since $J_{\beta}(\sigma)$ also depends on $w$, we first differentiate the map

$$
\sigma \rightarrow w(\sigma)
$$

We give the two theorems from [12] deriving the necessary conditions and briefly summarize the proofs.

Theorem 4.1. The map

$$
\sigma \in U_{K} \rightarrow w(\sigma) \in L^{2}(0, T ; V)
$$

is differentiable in the following sense:

$$
\frac{w(\sigma+\varepsilon \ell)-w(\sigma)}{\varepsilon} \rightarrow \xi \quad \text { in } L^{2}(0, T ; V)
$$

as $\varepsilon \rightarrow 0$, for $\sigma+\varepsilon \ell, \sigma \in U_{K}, \ell \in L^{\infty}(D)$. Furthermore, $\xi$ is a weak solution of

$$
\begin{aligned}
\xi_{t t}-\nabla(E \nabla \xi) & =0 & & \text { in } Q \\
\xi & =0 & & \text { on } \Sigma \times(0, T) \\
\frac{\partial \xi}{\partial \nu} & =0 & & \text { on } D \times\{z=0\} \times(0, T) \\
\frac{\partial \xi}{\partial \nu}+\sigma \xi & =-\ell w & & \text { on } \Gamma \times(0, T) \\
\xi=\xi_{t} & =0 & & \text { on } \Omega \times\{0\}
\end{aligned}
$$

where $w=w(\sigma)$ 
Proof: Using the notation of $w=w(\sigma), w^{\varepsilon}=w(\sigma+\varepsilon \ell)$ and noting the added regularity of $w$ and $w^{\varepsilon}$ from Proposition 3.2, we choose $\left(\frac{w^{\varepsilon}-w}{\varepsilon}\right)_{t}$ as test function in the weak solution form of the $\frac{w^{\varepsilon}-w}{\varepsilon}$ equation. On $Q_{s}=\Omega \times(0, s), \Gamma_{s}=\Gamma \times(0, s), 0<s \leq T$, we obtain

$$
\begin{aligned}
& \int_{Q_{s}}\left(\frac{w^{\varepsilon}-w}{\varepsilon}\right)_{t t}\left(\frac{w^{\varepsilon}-w}{\varepsilon}\right)_{t} d \Omega d t+\int_{Q_{s}} E \nabla\left(\frac{w^{\varepsilon}-w}{\varepsilon}\right) \cdot \nabla\left(\left(\frac{w^{\varepsilon}-w}{\varepsilon}\right)_{t}\right) d \Omega d t \\
& \quad+\int_{\Gamma_{s}} \sigma\left(\frac{w^{\varepsilon}-w}{\varepsilon}\right)\left(\frac{w^{\varepsilon}-w}{\varepsilon}\right)_{t} d \Gamma d t=-\int_{\Gamma_{s}} \ell w^{\varepsilon}\left(\frac{w^{\varepsilon}-w}{\varepsilon}\right)_{t} d \Gamma d t .
\end{aligned}
$$

Integrating by parts and using Cauchy-Schwarz one obtains

$$
\begin{aligned}
& \int_{\Gamma}\left(\left(\frac{w^{\varepsilon}-w}{\varepsilon}\right)_{t}\right)^{2}(s) d \Omega+\int_{\Omega}\left|\nabla\left(\frac{w^{\varepsilon}-w}{\varepsilon}\right)\right|^{2}(s) d \Omega+\int_{\Gamma} \sigma\left(\frac{w^{\varepsilon}-w}{\varepsilon}\right)^{2}(s) d \Omega \\
& \quad \leq C \int_{\Gamma_{s}}\left(w_{t}^{\varepsilon}\right)^{2} d \Gamma d t+C_{\delta} \int_{\Gamma}\left(w^{\varepsilon}\right)^{2}(s) d \Gamma+\int_{\Gamma_{s}}\left(\frac{w^{\varepsilon}-w}{\varepsilon}\right)^{2} d \Gamma+\delta \int_{\Gamma}\left(\frac{w^{\varepsilon}-w}{\varepsilon}\right)^{2}(s) d \Gamma .
\end{aligned}
$$

Using trace estimates and Poincaré's Inequality on the right hand side, we have

$$
\begin{aligned}
& \int_{\Omega}\left(\left(\frac{w^{\varepsilon}-w}{\varepsilon}\right)_{t}\right)^{2}(s) d \Omega+(1-\delta C) \int_{\Omega}\left|\nabla\left(\frac{w^{\varepsilon}-w}{\varepsilon}\right)\right|^{2}(s) d \Omega+\int_{\Gamma} \sigma\left(\frac{w^{\varepsilon}-w}{\varepsilon}\right)^{2}(s) d \Gamma \\
& \quad \leq C+\int_{0}^{s} \int_{\Omega}\left|\nabla\left(\frac{w^{\varepsilon}-w}{\varepsilon}\right)\right|^{2}(t) d \Omega d t .
\end{aligned}
$$

Finally, choosing $\delta$ small so that $1-\delta C>0$ and applying Gronwall's Inequality [6] gives

$$
\sup _{0 \leq d \leq T} \int_{\Omega}\left(\left(\frac{w^{\varepsilon}-w}{\varepsilon}\right)\right)_{t}^{2}(s) d \Omega+\int_{\Omega}\left|\nabla\left(\frac{w^{\varepsilon}-w}{\varepsilon}\right)\right|^{2}(s) d \Omega+\int_{\Gamma} \sigma\left(\frac{w^{\varepsilon}-w}{\varepsilon}\right)^{2}(s) d \Gamma \leq C_{1} .
$$

As in the proof of Theorem 3.1, this estimate implies

$$
\left\|\frac{w^{\varepsilon}-w}{\varepsilon}\right\|_{L^{2}\left(0, T ; V^{\prime}\right)} \leq C
$$

and on a subsequence, as $\varepsilon \rightarrow 0$,

$$
\begin{gathered}
\frac{w^{\varepsilon}-w}{\varepsilon} \rightarrow \xi \quad \text { in } L^{2}(0, T ; V) \\
\left(\frac{w^{\varepsilon}-w}{\varepsilon}\right)_{t t} \rightarrow \xi_{t t} \quad \text { in } L^{2}\left(0, T ; V^{\prime}\right) \\
\left(\frac{w^{\varepsilon}-w}{\varepsilon}\right)_{t} \rightarrow \xi_{t} \quad \text { in } L^{2}\left(0, T ; L^{2}(\Omega)\right) \\
\frac{w^{\varepsilon}-w}{\varepsilon} \rightarrow \xi \text { in } L^{2}\left(0, T ; L^{2}(\Gamma)\right) .
\end{gathered}
$$


Also $\xi$ satisfies (4.1) in the weak sense:

$$
\left\langle\xi_{t t}, v\right\rangle+B[\xi, v ; t]=-\int_{\Gamma} \ell w v(t) d \Gamma, \quad v \in V, \text { a.e. } t .
$$

Next, we state the necessary conditions:

Theorem 4.2. Given an optimal control $\sigma_{\beta}$ and a corresponding state $w_{\beta}=w\left(\sigma_{\beta}\right)$, there exists an adjoint solution $p$ in $L^{2}(0, T ; V)$, solving in a weak sense the adjoint system:

$$
\begin{array}{rlrl}
p_{t t}-\nabla(E \nabla p) & =\left(w_{\beta}-h\right) \chi_{G} \quad \text { in } Q \\
p & =0 & & \text { on } \Sigma \times(0, T) \\
\frac{\partial p}{\partial \nu} & =0 & & \text { on } D \times\{z=0\} \times(0, T) \\
\frac{\partial p}{\partial \nu}+\sigma_{\beta} p & =0 & & \text { on } \Gamma \times(0, T) \\
p=p_{t} & =0 & & \text { on } \Omega \times\{T\} .
\end{array}
$$

Furthermore,

$$
\sigma_{\beta}(x, y)=\min \left\{\left(\frac{1}{\beta} \int_{0}^{T} w_{\beta} p(x, y, u(x, y), t) d t\right)^{+}, K\right\}
$$

Proof: Since $\left(w_{\beta}-h\right) \chi_{G} \in L^{2}(Q)$, we can obtain the existence and uniqueness of the weak solution $p$ by arguments like in Theorem 3.1 .

Suppose $\sigma_{\beta}+\varepsilon \ell \in U_{K}$ for $\varepsilon$ small. Consider the directional derivative of $J$ with respect to $\sigma$ at $\sigma_{\beta}$ in the direction $\ell$ :

$$
\begin{aligned}
0 & \leq \lim _{\varepsilon \rightarrow 0^{+}} \frac{J_{\beta}\left(\sigma_{\beta}+\varepsilon \ell\right)-J_{\beta}\left(\sigma_{\beta}\right)}{\varepsilon} \\
& =\int_{G \times(0, T)} \xi\left(w_{\beta}-h\right) d G d t+\beta \int_{\Gamma} \sigma_{\beta} \ell d \Gamma \\
& =\int_{0}^{T}\left\langle p_{t t}, \xi\right\rangle d t+\int_{0}^{T} B[p, \xi ; t] d t+\beta \int_{\Gamma} \sigma_{\beta} \ell d \Gamma \\
& =-\int_{\Gamma \times(0, T)} \ell w_{\beta} p d \Gamma d t+\beta \int_{\Gamma} \sigma_{\beta} \ell d \Gamma \\
& =\int_{\Gamma} \ell(x, y)\left(-\int_{0}^{T} w_{\beta} p(x, y, u(x, y), t) d t+\beta \sigma_{\beta}(x, y)\right) d \Gamma
\end{aligned}
$$


by using the adjoint equation (4.3) and then using the equation (4.1) satisfied by $\xi$. Standard control arguments give

$$
\sigma_{\beta}(x, y)=\min \left\{\left(\frac{1}{\beta} \int_{0}^{T} w_{\beta} p(x, y, u(x, y), t) d t\right)^{+}, K\right\} .
$$

\section{Uniqueness of the Optimal Control}

Uniqueness was not addressed in [12]; we now prove the following result:

Theorem 5.1. For $T$ sufficiently small, the solution of the optimality system (OS) is unique:

$$
\begin{aligned}
& w_{t t}-\nabla(E \nabla w)=f \quad \text { in } Q \\
& p_{t t}-\nabla(E \nabla p)=(w-h) \chi_{G} \text { in } Q \\
& w=p=0 \quad \text { on } \Sigma \times(0, T) \\
& \frac{\partial w}{\partial \nu}=\frac{\partial p}{\partial \nu}=0 \quad \text { on } D \times\{z=0\} \times(0, T) \\
& w=g_{1}, \quad w_{t}=g_{2} \quad \text { on } \Omega \times\{0\} \\
& p=p_{t}=0 \quad \text { on } \Omega \times\{T\} \\
& \frac{\partial w}{\partial \nu}+\min \left\{\frac{1}{\beta}\left(\int_{0}^{T} w p(x, y, u(x, y), t) d t\right)^{+}, K\right\} w=0 \quad \text { on } \Gamma \times(0, T) \\
& \frac{\partial p}{\partial \nu}+\min \left\{\frac{1}{\beta}\left(\int_{0}^{T} w p(x, y, u(x, y), t) d t\right)^{+}, K\right\} p=0 \quad \text { on } \Gamma \times(0, T) \text {. }
\end{aligned}
$$

Proof. Suppose $w, p$ and $\bar{w}, \bar{p}$ are solutions of (OS) system. Let $\hat{w}=w-\bar{w}, \hat{p}=p-\bar{p}$. Consider the $\hat{w}$ equation on $\Omega \times(0, t), 0<t \leq T$, and obtain

$$
\int_{\Omega}\left[\left(\hat{w}_{t}(t)\right)^{2}+|\nabla \hat{w}(t)|^{2}\right] d \Omega+\int_{\Gamma} \sigma \hat{w}(t)^{2} d \Gamma=-2 \int_{0}^{t} \int_{\Gamma}(\sigma-\bar{\sigma}) \bar{w} \hat{w}_{t} d \Gamma d \tau
$$

where

$$
\sigma(x, y)=\min \left\{\frac{1}{\beta}\left(\int_{0}^{T} w p(x, y, u(x, y), t) d t\right)^{+}, K\right\}
$$

and

$$
\bar{\sigma}(x, y)=\min \left\{\frac{1}{\beta}\left(\int_{0}^{T} \bar{w} \bar{p}(x, y, u(x, y), t) d t\right)^{+}, K\right\}
$$


Similarly on $\Omega \times(t, T), 0<t \leq T$, in the adjoint case, we obtain

$$
\begin{aligned}
& \int_{\Omega}\left[\left(\hat{p}_{t}(t)\right)^{2}+|\nabla \hat{p}(t)|^{2}\right] d \Omega+\int_{\Gamma} \sigma(\hat{p}(t))^{2} d \Gamma \\
& \quad=-2 \int_{0}^{T} \int_{G} \hat{w} \hat{p}_{t} d G d \tau+2 \int_{t}^{T} \int_{\Gamma}(\sigma-\bar{\sigma}) \bar{p} \hat{p}_{t} d \Gamma d \tau .
\end{aligned}
$$

Integration by parts gives

$$
\int_{t}^{T} \int_{\Gamma}(\sigma-\bar{\sigma}) \bar{p} \hat{p}_{t} d \Gamma d \tau=-\int_{t}^{T} \int_{\Gamma}(\sigma-\bar{\sigma}) \bar{p} t \hat{p} d \Gamma d \tau-\int_{\Gamma}(\sigma-\bar{\sigma}) \bar{p} \hat{p}(t) d \Gamma
$$

Continuing to estimate, we have

$$
\begin{aligned}
& \int_{t}^{T} \int_{\Gamma}\left|\sigma-\bar{\sigma}\left\|\bar{p}_{t}|\| \hat{p}| d \Gamma d \tau\right.\right. \\
\leq & \frac{1}{2} \int_{t}^{T} \int_{\Gamma}(\sigma-\bar{\sigma})^{2} d \Gamma d \tau+\frac{1}{2} \int_{t}^{T} \int_{\Gamma}\left|\bar{p}_{t}\right|^{2}|\hat{p}|^{2} d \Gamma d \tau \\
\leq & C \int_{t}^{T} \int_{\Gamma} \int_{0}^{T} \bar{w}^{2} \hat{p}^{2}+p^{2} \hat{w}^{2} d \tau_{1} d \Gamma d \tau+\frac{1}{2} \int_{t}^{T}\left(\int_{\Gamma}\left(\bar{p}_{t}\right)^{4} d \Gamma\right)^{\frac{1}{2}}\left(\int_{\Gamma} \hat{p}^{4} d \Gamma\right)^{\frac{1}{2}} d \tau \\
\leq & C \int_{t}^{T} \int_{0}^{T}\left(\int_{\Gamma} \bar{w}^{4}\left(\tau_{1}\right) d \Gamma\right)^{\frac{1}{2}}\left(\int_{\Gamma} \hat{p}^{4}\left(\tau_{1}\right) d \Gamma\right)^{\frac{1}{2}} d \tau_{1} d \tau \\
& +\int_{t}^{T} \int_{0}^{T}\left(\int_{\Gamma} p^{4}\left(\tau_{1}\right) d \Gamma\right)^{\frac{1}{2}}\left(\int_{\Gamma} \hat{w}^{4}\left(\tau_{1}\right) d \Gamma\right)^{\frac{1}{2}} d \tau_{1} d \tau+C_{2} \int_{t}^{T}\left(\int_{\Gamma} \hat{p}^{4} d s\right)^{\frac{1}{2}} d \tau
\end{aligned}
$$

where we used that $\bar{p}$ satisfies the additional regularity like in Proposition 3.2 and

$$
\bar{p}_{t}(t) \in H^{1}(\Omega) \hookrightarrow L^{4}(\Gamma) .
$$

If a similar estimate on $\bar{w}, p$ is used, one obtains

$$
\int_{t}^{T}\left|\sigma-\bar{\sigma}\left\|\bar{p}_{t}\right\| \hat{p}\right| d \Gamma d \tau \leq C T \sup _{0 \leq t \leq T}\left(\|\nabla \hat{p}(t)\|_{L^{2}(\Omega)}^{2}+\|\nabla \hat{w}(t)\|_{L^{2}(\Omega)}\right) .
$$

For $\epsilon>0$, estimate the other type of boundary term in (5.3)

$$
\begin{aligned}
& \int_{\Gamma}|\sigma-\bar{\sigma}||\bar{p}||\hat{p}|(t) d \Gamma \\
\leq & C_{\epsilon} \int_{\Gamma}(\sigma-\bar{\sigma})^{2} d \Gamma+\epsilon \int_{\Gamma}|\bar{p}|^{2}|\hat{p}|^{2}(t) d \Gamma \\
\leq & \hat{C}_{\epsilon} T \sup _{0 \leq t \leq T}\left[\|\nabla \hat{p}(t)\|_{L^{2}(\Omega)}^{2}+\|\nabla \hat{w}(t)\|_{L^{2}(\Omega)}^{2}\right]+\epsilon\|\nabla \hat{p}(t)\|_{L^{2}(\Omega)}^{2},
\end{aligned}
$$


using similar imbeddings involving $L^{4}(\Gamma)$.

Taking the supremum over $t$ in the adjoint estimate, gives

$$
\begin{aligned}
& \sup _{0 \leq t \leq T}\left(\left\|\hat{p}_{t}(t)\right\|_{L^{2}(\Omega)}^{2}+\|\nabla \hat{p}(t)\|_{L^{2}(\Omega)}^{2}+\int_{\Gamma} \sigma \hat{p}^{2}(t) d \Gamma\right) \\
& \leq \bar{C}_{\epsilon} T \sup _{0 \leq t \leq T}\left(\|\nabla \hat{p}(t)\|_{L^{2}(\Omega)}^{2}+\|\nabla \hat{w}(t)\|_{L^{2}(\Omega)}^{2}+\left\|\hat{w}_{t}(t)\right\|_{L^{2}(\Omega)}^{2}+\left\|\hat{p}_{t}(t)\right\|_{L^{2}(\Omega)}^{2}\right) \\
& +\epsilon \sup _{0 \leq t \leq T}\left(\|\nabla \hat{p}(t)\|_{L^{2}(\Omega)}^{2}+\|\nabla \hat{w}(t)\|_{L^{2}(\Omega)}^{2}\right) .
\end{aligned}
$$

We have a similar estimate for $\hat{w}$.

Adding the supremum estimates for $\hat{p}, \hat{w}$ and taking $\epsilon$ small enough and then $T$ small so that

$$
2\left(\bar{C}_{\epsilon} T+\epsilon\right)<1
$$

we obtain

$$
\sup _{0 \leq t \leq T}\left(\left\|\hat{p}_{t}(t)\right\|_{L^{2}(\Omega)}^{2}+\|\nabla \hat{p}(t)\|_{L^{2}(\Omega)}^{2}++\left\|\hat{w}_{t}(t)\right\|_{L^{2}(\Omega)}^{2}+\|\nabla \hat{w}(t)\|_{L^{2}(\Omega)}^{2}\right) \leq 0
$$

which gives uniqueness. $\quad$ a

\section{Approximate Solution of the Parameter Identification Problem}

Having solved the optimal control problem, we now use a sequence of optimal controls to approximate the solution of the parameter identification problem. We view $h$ as an observation of the solution $w$ on $G \times(0, T)$ and seek to identify a reflection coefficient $\sigma_{0}$ such that the solution $w=w\left(\sigma_{0}\right)$ satisfies

$$
\left.w\right|_{G \times(0, T)}=h
$$

Assuming the existence of a solution of this inverse problem, we will approximate $\sigma_{0}$ by the optimal control $\sigma_{\beta}$ as $\beta$ gets small.

Theorem 6.1. Suppose the inverse problem has a solution, i.e. there exists $\sigma^{*} \in U_{K}$ such that $w^{*}=w\left(\sigma^{*}\right)$ satisfies $w^{*}=h$ a.e. on $G \times(0, T)$. Then there exists $0 \leq \sigma_{0} \leq K$ such that on a subsequence of $\beta \rightarrow 0$,

$$
\begin{aligned}
\sigma_{\beta} & \rightarrow \sigma_{0} \quad \text { in } L^{2}(\Gamma) \\
w_{\beta}=w\left(\sigma_{\beta}\right) & \rightarrow w_{0} \quad \text { in } L^{2}(0, T ; V)
\end{aligned}
$$

and

$$
w_{0}=h \quad \text { a.e. on } G \times(0, T)
$$


Proof. Notice that $\sigma^{*} \in U_{K}$ and let $\sigma_{\beta}$ be an optimal control that minimizes $J_{\beta}(\sigma)$. Then we have

$$
J_{\beta}\left(\sigma_{\beta}\right) \leq J_{\beta}\left(\sigma^{*}\right)
$$

and

$$
\frac{1}{2} \int_{G \times(0, T)}\left(w_{\beta}-h\right)^{2} d G d t+\frac{\beta}{2} \int_{\Gamma} \sigma_{\beta}^{2} d \Gamma \leq \frac{\beta}{2} \int_{\Gamma}\left(\sigma^{*}\right)^{2} d \Gamma
$$

Using the above a priori estimates on $w_{\beta}$, which are independent of $\beta$, for a (sub)sequence $\beta \rightarrow 0$, we have

$$
\begin{aligned}
w_{\beta} & \rightarrow w_{0} \quad \text { in } L^{2}(0, T ; V) \\
\left(w_{\beta}\right)_{t t} & \rightarrow\left(w_{0}\right)_{t t} \quad \text { in } L^{2}\left(0, T ; V^{\prime}\right) \\
\left(w_{\beta}\right)_{t} & \rightarrow\left(w_{0}\right)_{t} \quad \text { in } L^{2}\left(0, T ; L^{2}(\Omega)\right) \\
\sigma^{\beta} & \rightarrow \sigma_{0} \quad \text { in } L^{2}(\Gamma)
\end{aligned}
$$

and

$$
w_{0}=w\left(\sigma_{0}\right)
$$

Letting $\beta \rightarrow 0$ in (6.1), we also obtain

$$
\int_{G \times(0, T)}\left(w_{0}-h\right)^{2} d G d t=0 .
$$

Thus $w_{0}$ has the desired property, i.e., $w=h$ a.e. on $G \times(0, T)$, and $\sigma_{0}$ is the coefficient that is "identified". If the solution of the inverse problem, $\sigma^{*}$, is unique, then $\sigma_{0}=\sigma^{*}$.

\section{Discussion}

Optimal control techniques applied to an acoustic wave equation provide a systematic method to approximate a boundary reflection coefficient, $\sigma$, that yields a solution consistent with the actual observations. By the same procedure, we also have an explicit characterization of the approximations $\sigma_{\beta}$.

In the previous discussion we did not take into account causality effects that are due to the finite speed of propagation of signals in hyperbolic systems. Indeed, if we suppose that: (i) at $u=u_{t}=0$ at $t=0$, (ii) the source is localized, and (iii) the receptors are far from the source, then there will be a time interval during which both the measured signal and the model solution are zero at the receptor location. Thus, for that time interval (whose length will depend on the signal speed and the actual geometric configuration), the first 
term in the functional (1.3) is zero, which means that the minimum of $J$ is realized for $\sigma=0$, independently of the value of the actual reflection coefficient at the boundary.

In order to obtain meaningful results, the time interval should be large enough to allow signals reflected by the boundary to actually reach the receptors and overcome the spurious threshold effects. Of course, this may hamper the uniqueness result which, as noted, is valid only for short times.

Another source of meaningless results is related to model inadequacies and/or the presence of noise. Indeed, suppose the model does not predict any (reflected) signal at the receptor, i.e. $u=0$, while the actual measured signal, $h$, is different from zero (or vice-versa). Again the minimizer is given by the (most likely wrong) result $\sigma=0$, independently of its actual value or functional form. This spurious result occurs because our method assumes the model is completely and correctly known. The knowledge of the model is paramount to this parameter identification method and any model imperfection will be reflected sometimes in the form of severe anomalies - in the identified parameters.

Finally, we expect the quality of the recovery to increase with the amount and quality of the available information. In other words, the larger $G$ is the better the results are expected to become. However this relationship cannot be expressed by a simple monotonic dependence.

\section{Acknowledgments}

S.L. and V.P. acknowledge partial support from the US Department of Energy, Office of Basic Energy Sciences, under contract No. AC05-96OR22464 with Lockheed Martin Energy Research Corporation. J.Y. is supported in part by the NNSF of China, the Chinese State Education Commission Science Foundation, and the Trans-Century Training Programme Foundation for Talents of the State Education Commission of China.

\section{REFERENCES}

1. H.T. Banks and K. Kunisch, Estimation Techniques for Distributed Parameter Systems, Birkhäuser, Boston, 1989.

2. V. Barbu and N. H. Pavel, Determining the acoustic impedance in the 1-D wave equation via an optimal control problem, SIAM J. Optimal Control 35 (1997), 1544-1556.

3. B.P. Belinskiy, J.P. Dauer, C. Martin, and M.A. Shubov, On controllability of an oscillating continuum with a viscous damping, preprint.

4. J. Chavent, On parameter idenitifiability, Proceedings of the 7 th IFAC Symposium on Identification and System Parameter Estimations, Pergamon Press, York, 1985, pp. 531-36.

5. J. D. Craig and C. Brown, Inverse Problems in Astronomy, Adam Hilger Ltd., Bristol and Boston, 1986.

6. L.C. Evans, Partial Differential Equations Vol. 3B (1993), Berkeley Math Lecture Notes, Berkeley.

7. K.D. Graham and D.L. Russell, Boundary value control of the wave equation in a spherical region, SIAM Journal on Control 13 (1975), 174-196.

8. C.W. Groetsch, Inverse Problems in the Mathematical Sciences, Vieweg, Braunschweig, Wiesbaden, 1993.

9. F. James and M. Sepulveda, Parameter identification for a model of chromatographic column, Inverse Problems 10 (1994), 367-385. 
10. I. Lasiecka and R. Triggiani, Differential and algebraic Riccati equations with applications to boundary/point control problems: Continuous theory and approximation theory, Lecture Notes in Control and Information Sciences, vol. 164, Springer-Verlag, New York, 1991.

11. I. Lasiecka and R. Triggiani, Sharp regularity theory for second order hyperbolic equations of Neumann type Part I - nonhomogeneous data, Annali di Mat. Pura ed Appl. CLVII (1990), 285-367.

12. S. Lenhart, V. Protopopescu, and J. Yong, Optimal control of a reflection boundary coefficient in an acoustic wave equation, Applicable Analysis, to appear.

13. S. Lenhart, V. Protopopescu, and J. Yong, Solving inverse problems of identification type by optimal control methods, Proceedings of the International Conference on Advances of Nonlinear Dynamics near the millenium, ANDM'97, 1997, to appear.

14. S. Lenhart and D.G. Wilson, Optimal control of a heat transfer problem with convective boundary condition, J. Optimization Theory and Applications 79 (1993), 581-597.

15. M. Liang, Bilinear optimal control of a wave equation, preprint, University of Tennessee, Knoxville.

16. J.-L. Lions and E. Magenes, Non-Homogeneous Boundary Value Problems, vols. 1-3, Springer Verlag, New-York, 1972.

17. J.-L. Lions, Optimal Control of Systems Governed by Partial Differential Equations, Springer Verlag, New York, 1971.

18. J.-L. Lions, Exact controllability, stabilization, and perturbation for distributed systems, SIAM Review $30(1988), 1-68$.

19. J.-L. Lions, Least regret controls for distributed systems, (in French), C.R. Acad. Sci. Paris 315 (1992), Série I, 1253-1257.

20. J.-L. Lions, Some Methods in the Mathematical Analysis of Systems and Their Controls, Science Press, Beijing and Gordon \& Breach, New York, 1981.

21. J-P. Puel and M. Yamamoto, Applications of exact controllability to some inverse hyperbolic problems, (in French), C.R. Acad. Sci. Paris 320 (1995), Série I, 1171-1176.

22. J.-P. Puel and M. Yamamoto, On a global estimate in a linear inverse hyperbolic problem, Inverse Problems 12 (1996), 995-1002.

23. D.L. Russell, A unified boundary controllability theory for hyperbolic and parabolic partial differential equations, Studies in Applied Mathematics LII (1973), 189-211.

24. D.L. Russell, Boundary value control of the higher dimensional wave equation, SIAM J. on Control 9 (1971), 29-42.

25. D.L. Russell, Optimal regulation of linear symmetric hyperbolic systems with finite dimensional controls, SIAM J. on Control 4 (1966), 276-294.

26. D.L. Russell, On boundary-value controllability of linear symmetric hyperbolic systems, Mathematical Theory of Control, Academic Press, New York, 1967, pp. 312-321.

27. J. Simon, Compact sets in the space $L^{p}(0, T ; B)$, Annali di Matematica Pura ed Applicata CXLVI (1987), 65-96.

28. A. N. Tikhonov and V. Y. Arsenin, Solutions of Ill-Posed Problems, John Wiley, New York, 1977.

29. M. Yamamoto, Stability, reconstruction formula, and regularization for an inverse source hyperbolic problem by a control method, Inverse Problems 11 (1995), 481-496. 